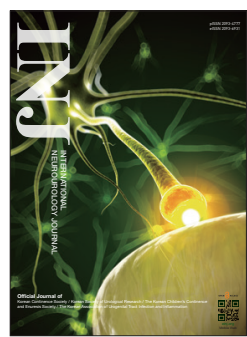

\title{
Development of Decision Support Formulas for the Prediction of Bladder Outlet Obstruction and Prostatic Surgery in Patients With Lower Urinary Tract Symptom/Benign Prostatic Hyperplasia: Part I, Development of the Formula and its Internal Validation
}

\author{
Min Soo Choo ${ }^{1}$, Changwon $\mathrm{Yoo}^{2}$, Sung Yong Cho ${ }^{3}$, Seong Jin Jeong ${ }^{4}$, Chang Wook Jeong ${ }^{5}$, Ja Hyeon $\mathrm{Ku}^{5}$, Seung-June $\mathrm{Oh}^{5}$ \\ ${ }^{1}$ Department of Urology, Hallym University Dongtan Sacred Heart Hospital, Hwaseong, Korea \\ ${ }^{2}$ Department of Biostatistics, Robert-Stempel College of Public Health \& Social Work, Florida International University, Miami, FL, USA \\ ${ }^{3}$ Department of Urology, Seoul Metropolitan Government-Seoul National University Boramae Medical Center, Seoul, Korea \\ ${ }^{4}$ Department of Urology, Seoul National University Bundang Hospital, Seongnam, Korea \\ ${ }^{5}$ Department of Urology, Seoul National University Hospital, Seoul, Korea
}

Purpose: As the elderly population increases, a growing number of patients have lower urinary tract symptom (LUTS)/benign prostatic hyperplasia (BPH). The aim of this study was to develop decision support formulas and nomograms for the prediction of bladder outlet obstruction (BOO) and for BOO-related surgical decision-making, and to validate them in patients with LUTS/BPH.

Methods: Patient with LUTS/BPH between October 2004 and May 2014 were enrolled as a development cohort. The available variables included age, International Prostate Symptom Score, free uroflowmetry, postvoid residual volume, total prostate volume, and the results of a pressure-flow study. A causal Bayesian network analysis was used to identify relevant parameters. Using multivariate logistic regression analysis, formulas were developed to calculate the probabilities of having BOO and requiring prostatic surgery. Patients between June 2014 and December 2015 were prospectively enrolled for internal validation. Receiver operating characteristic curve analysis, calibration plots, and decision curve analysis were performed.

Results: A total of 1,179 male patients with LUTS/BPH, with a mean age of 66.1 years, were included as a development cohort. Another 253 patients were enrolled as an internal validation cohort. Using multivariate logistic regression analysis, 2 and 4 formulas were established to estimate the probabilities of having $\mathrm{BOO}$ and requiring prostatic surgery, respectively. Our analysis of the predictive accuracy of the model revealed area under the curve values of 0.82 for BOO and 0.87 for prostatic surgery. The sensitivity and specificity were $53.6 \%$ and $87.0 \%$ for BOO, and $91.6 \%$ and $50.0 \%$ for prostatic surgery, respectively. The calibration plot indicated that these prediction models showed a good correspondence. In addition, the decision curve analysis showed a high net benefit across the entire spectrum of probability thresholds.

Conclusions: We established nomograms for the prediction of BOO and BOO-related prostatic surgery in patients with LUTS/BPH. Internal validation of the nomograms demonstrated that they predicted both having BOO and requiring prostatic surgery very well.

Keywords: Prostatic Hyperplasia; Decision Support Systems, Clinical; Nomograms; Urinary Bladder Neck Obstruction; Prostatectomy

- Fund/Grant Support: This study was supported by grant from the Seoul National University Hospital Research Fund (34-2014-0100).

- Research Ethics: This study was approved by the Institutional Review Board of Seoul National University Hospital (approval number: H-1406119-591).

- Conflict of Interest: MSC, a member of the Editorial Board of INJ, is the first author of this article. However, he played no role whatsoever in the editorial evaluation of this article or the decision to publish it. No potential conflict of interest relevant to this article was reported.

Corresponding author: Seung-June Oh (iD http://orcid.org/0000-0002-0322-3539 Department of Urology, Seoul National University Hospital, 101 Daehak-ro, Jongno-gu, Seoul 03080, Korea

E-mail: sjo@snu.ac.kr / Tel +82-2-2072-2406 / Fax: +82-2-742-4665

Submitted: March 22, 2017 / Accepted after revision: April 7, 2017 (c) (1) This is an Open Access article distributed under the terms of the Creative Commons Attribution Non-Commercial License (http://creativecommons.org/licenses/by-nc/4.0/) which permits unrestricted non-commercial use, distribution, and reproduction in any medium, provided the original work is properly cited. 


\section{INTRODUCTION}

As the elderly population increases, a growing number of patients have lower urinary tract symptom (LUTS)/benign prostatic hyperplasia (BPH) [1]. The treatment of BPH includes watchful waiting, medical management, and surgical treatment. Medication has been widely used in patients with mild to borderline symptoms. The guidelines of the European Urological Association and the American Urological Association indicate that surgical treatment is necessary when patients have urinary retention, renal insufficiency, recurrent urinary tract infections, bladder stone, or gross hematuria due to an enlarged prostate $[2,3]$.

However, most patients with LUTS/BPH we encounter in clinical practice do not fall under the above absolute indications. Surgery is additionally needed when patients do not experience adequate relief from LUTS with conservative or medical treatment [2]. This relative surgical indication usually reflects the subjective judgment of the surgeon, and/or patients degree of compliance and preference for surgery [3]. In real clinical situations, the decision is not simple. It is sometimes difficult to make a clear decision about the treatment of those who do not have an absolute indication for prostatic surgery. Therefore, the development of objective therapeutic guidance is necessary.

LUTS/BPH is a progressive disease that can eventually cause irreversible changes in bladder function, especially if bladder outlet obstruction (BOO) is already present. Therefore, when $\mathrm{BOO}$ is evident in patients with bothersome LUTS, surgical treatment should be considered. A pressure-flow study (PFS) is currently considered the gold standard for diagnosing BOO; however, it is an invasive and time-consuming procedure. Noninvasive or less invasive modalities have not been fully validated for clinical use.

At the Seoul National University Hospital (SNUH), we have a large urodynamic study database that has been constructed over the last 13 years. Based on this, we aimed to develop decision support formulas and nomograms predicting the probabilities of having $\mathrm{BOO}$ and requiring $\mathrm{BOO}$-related prostatic surgery in patients with LUTS/BPH. We also attempted to validate these formulas and nomograms in a prospective cohort of patients with LUTS/BPH.

\section{MATERIALS AND METHODS}

\section{Patients and Evaluation}

We have constructed a urodynamic study database at SNUH starting in 2004. From this large database consisting of 11,437 consecutive patients between October 2004 and May 2014, LUTS/BPH patients aged 45 or over were extracted. LUTS involving the following conditions were excluded to construct pure a LUTS/BPH patient dataset (the development cohort): urethral stricture, bladder stone, genitourinary infection or inflammation, genitourinary malignancy, genitourinary radiation, urinary diversion, or neurogenic bladder. We additionally excluded 309 patients who had previously undergone prostatic surgery. We ultimately retrieved 2,732 patients with pure LUTS/ $\mathrm{BPH}$.

Clinical parameters were obtained in routine, real clinical practice settings, with careful history-taking, a physical examination including a digital rectal examination, the International Prostate Symptom Score (IPSS), laboratory tests including urinalysis and serum prostate-specific antigen (PSA) levels, and a voiding diary. After free uroflowmetry (UFM), postvoid residual (PVR) volume was measured using an ultrasound bladder scanner (BVI-3000 BladderScan, Verathon, WA, USA or BioCon-500, Mcube Technology, Seoul, Korea) in the supine position. The IPSS storage subscore was the sum of IPSS questions 2,4 , and 7 , and the voiding subscore was the sum of IPSS questions $1,3,5$, and 6 . UFM results were accepted only when the voided volume was over $120 \mathrm{~mL}$. Prostatic biopsies were performed to exclude cancer when the serum PSA level was elevated ( $>4 \mathrm{ng} / \mathrm{mL}$ ) or when a digital rectal examination was suspicious for prostate cancer. Total prostate volume (TPV) was calculated using the prolate ellipsoid formula $(\pi / 6 \times$ height $\times$ width $\times$ length) based on transrectal ultrasonography (TRUS) measurements in 3 dimensions. A PFS to determine whether BOO was present was performed in our Urodynamic Suite using a multichannel urodynamic machine (UD-2000 or Solar, MMS, Enschede, the Netherlands), as previously described [4]. We strictly followed the International Continence Society standards in performing urodynamic studies [5]. BOO was defined as a $\mathrm{BOO}$ index $\geq 40$; the $\mathrm{BOO}$ index was determined by the formula of detrusor pressure at the maximal flow rate (PdetQmax) $-2 \times$ maximal flow rate (Qmax) in the PFS [6]. Of the 2,732 patients, any patient who was missing 1 or more of the following clinical variables was excluded: IPSS, voided volume for UFM of at least $120 \mathrm{~mL}$, PVR volume, TRUS-measured 
TPV, PFS, and information regarding surgery.

The electronic medical records of all patients were thoroughly reviewed to exclude any patients who fulfilled the exclusion criteria. Ultimately, a database of pure LUTS/BPH patients with complete clinical variables was obtained as the development cohort for developing both formulas (for having BOO and for undergoing prostatic surgery). The need for prostatic surgery was based on the clinical judgment of urologists, not the actual performance of surgery. Therefore, if patients did not agree to undergo prostatic surgery when clinicians recommended it, we still classified these patients as requiring prostatic surgery. The prostatic surgery techniques for LUTS/BPH included open prostatectomy, transurethral resection of the prostate, transurethral incision of the prostate, laser photoselective vaporization of the prostate, and holmium laser enucleation of the prostate.

Separately from the development cohort, we prospectively enrolled consecutive LUTS/BPH patients with the same inclusion and exclusion criteria between June 2014 and December 2015 at SNUH to construct an internal validation cohort. This study was approved by the Institutional Review Board of SNUH (approval number: H-1406-119-591).

\section{Statistical Analysis}

Causal Bayesian network (CBN) analysis was performed to identify clinically relevant variables related to $\mathrm{BOO}$ and prostatic surgery. Using ovals to represent variables and arcs for their plausible causal relationships, $\mathrm{CBN}$ visually presents firstand second-degree relationships among the nomograms of clinical parameters for $\mathrm{BOO}$ and prostatic surgery. We used Banjo ver. 2.2.0 (Duke University, Durham, NC, USA; noncommercially available at https://users.cs.duke.edu/ amink/ software/banjo/) [7,8] to analyze the collected data and to develop the best-fitting $\mathrm{CBN}$ model. The $\mathrm{CBN}$ model identified the most relevant clinical variables and predicted the probability of a subject having $\mathrm{BOO}$ and the probability of a subject requiring prostatic surgery. We additionally used multivariate logistic regression analysis incorporating the relevant clinical variables to build formulas to calculate the probabilities of having $\mathrm{BOO}$ and requiring prostatic surgery.

To evaluate the predictions based on the calculated probabilities, receiver operating characteristic curve analysis was applied to calculate the area under the curve (AUC) of the prediction, with AUCs of 0.70-0.79 considered to indicate acceptable discrimination, $0.80-0.89$ to indicate excellent discrimination, and $>0.90$ to indicate outstanding discrimination [9]. To assess cali- bration, calibration plots were generated to visualize the agreement between the predicted probability of both having BOO and requiring prostatic surgery, and the actual fact of both having $\mathrm{BOO}$ and undergoing prostatic surgery. Decision curve analysis was used to assess the clinical usefulness of each prediction model by quantifying the net benefits achieved by making decisions at each probability threshold [10]. Sensitivity, specificity, the positive predictive value, and the negative predictive value were obtained and reported.

Two-sided P-values $<0.05$ were considered to indicate statistical significance. All statistical analyses were performed using R 3.3.1 (R Foundation for Statistical Computing, Vienna, Austria; http://www.R-project.org), and the IBM SPSS Statistics ver. 23.0 (IBM Co., Armonk, NY, USA).

\section{RESULTS}

\section{Patient Demographics}

A total of 1,179 patients with LUTS/BPH were included as the development cohort for the prediction models. The mean age of the patients was 66.1 years. The average TPV was $48.2 \mathrm{~mL}$, and the mean BOO index was 33.4 (Table 1). Among the patients who underwent a PFS for LUTS/BPH, prostatic surgery was recommended to 746 patients $(63.3 \%)$ by urologists. However, 616 patients (52.2\%) were found to have actually undergone surgery.

\section{Development of a Prediction Model}

Several clinically important parameters used for establishing the formulas were selected using a CBN analysis. TPV, Qmax, and PVR had a first-degree relationship with BOO. PSA and transitional zone volume (TZV) of the prostate had a seconddegree relationship with BOO. In addition, TPV and the BOO index had a first-degree relationship with prostatic surgery. The IPSS voiding subscore, PVR, Qmax of free flow, PSA, and TZV had a second-degree relationship with prostatic surgery (Fig. 1). Serum PSA level was significantly related to TPV; therefore, it was omitted from the final parameters, and the rest of the parameters were included to develop the formula.

In the multivariate logistic regression analysis, age, Qmax, PVR, and TPV were identified as independently significant variables associated with $\mathrm{BOO}$ (Table 2). In addition, age, Qmax, PVR, TPV, the IPSS voiding subscore, the IPSS storage subscore, the IPSS quality of life, and the BOO index were identified as independently significant variables associated with re- 
Table 1. Characteristics and comparison of patients' demographic variables between the development cohort and the internal validation cohort

\begin{tabular}{|c|c|c|c|}
\hline Characteristic & Development $(\mathrm{n}=1,179)$ & Internal validation $(\mathrm{n}=253)$ & P-value \\
\hline Age (yr) & $66.1 \pm 7.2$ & $69.1 \pm 6.8$ & $<0.001$ \\
\hline $\begin{array}{l}\text { IPSS } \\
\text { Voiding subscore } \\
\text { Storage subscore } \\
\text { Quality of life }\end{array}$ & $\begin{array}{r}17.8 \pm 7.8 \\
10.6 \pm 5.5 \\
7.0 \pm 3.4 \\
3.9 \pm 1.1\end{array}$ & $\begin{array}{r}17.3 \pm 8.0 \\
10.6 \pm 5.5 \\
7.1 \pm 3.4 \\
3.8 \pm 1.3\end{array}$ & $\begin{array}{l}0.244 \\
0.200 \\
0.539 \\
0.293\end{array}$ \\
\hline Total prostate volume $(\mathrm{mL})$ & $48.2 \pm 27.5$ & $60.7 \pm 31.0$ & $<0.001$ \\
\hline Transitional zone volume $(\mathrm{mL})$ & $23.7 \pm 21.4$ & $37.3 \pm 38.7$ & $<0.001$ \\
\hline Prostate-specific antigen (ng/mL) & $2.63 \pm 3.41$ & $3.4 \pm 6.7$ & 0.160 \\
\hline $\begin{array}{l}\text { Uroflowmetry parameters } \\
\text { Maximal flow rate }(\mathrm{mL} / \mathrm{sec}) \\
\text { Voided volume }(\mathrm{mL}) \\
\text { Postvoid residual volume }(\mathrm{mL})\end{array}$ & $\begin{array}{c}12.0 \pm 5.1 \\
234.5 \pm 106.1 \\
57.1 \pm 79.2\end{array}$ & $\begin{array}{r}11.7 \pm 4.7 \\
226.8 \pm 91.2 \\
63.9 \pm 31.0\end{array}$ & $\begin{array}{l}0.296 \\
0.782 \\
0.199\end{array}$ \\
\hline $\begin{array}{l}\text { Urodynamic study parameter } \\
\text { First desire }(\mathrm{mL}) \\
\text { Normal desire }(\mathrm{mL}) \\
\text { Strong desire }(\mathrm{mL}) \\
\text { MCC }(\mathrm{mL}) \\
\text { IDC } \\
\text { PdetQmax } \\
\text { Maximal flow rate of PFS } \\
\text { BOOI } \\
\text { BCI }\end{array}$ & $\begin{array}{c}205.4 \pm 91.5 \\
287.1 \pm 109.5 \\
373.3 \pm 108.3 \\
375.3 \pm 117.6 \\
298(25.3) \\
52.5 \pm 20.7 \\
9.1 \pm 4.6 \\
33.4 \pm 23.6 \\
98.1 \pm 27.1\end{array}$ & $\begin{array}{c}191.5 \pm 94.9 \\
206.5 \pm 97.5 \\
372.3 \pm 108.9 \\
328.0 \pm 145.3 \\
115(45.5) \\
54.1 \pm 22.4 \\
7.9 \pm 3.7 \\
38.9 \pm 25.1 \\
93.9 \pm 30.5\end{array}$ & $\begin{array}{r}0.647 \\
<0.001 \\
0.835 \\
<0.001 \\
<0.001 \\
0.213 \\
0.002 \\
0.001 \\
0.123\end{array}$ \\
\hline Decision to perform prostatic surgery & $746(63.3)$ & $201(79.4)$ & $<0.001$ \\
\hline Actually underwent prostatic surgery & $616(52.2)$ & $179(70.8)$ & $<0.001$ \\
\hline
\end{tabular}

Values are presented as mean \pm standard deviation or number (\%).

IPSS, International Prostate Symptom Score; voiding subscore, sum of questions 1, 3, 5, and 6 of the IPSS; storage subscore, sum of questions 2, 4, and 7 of the IPSS; quality of life, score of question 8 of the IPSS; MCC, maximal cystometric capacity; IDC, involuntary detrusor contraction; PdetQmax, detrusor pressure at the maximal flow rate; PFS, pressure-flow study; BOOI, bladder outlet obstruction index; BCI, bladder contractility index.

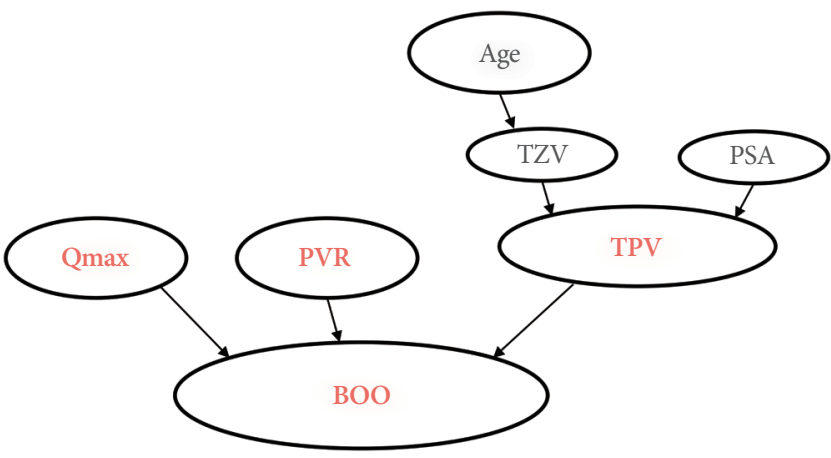

(A)

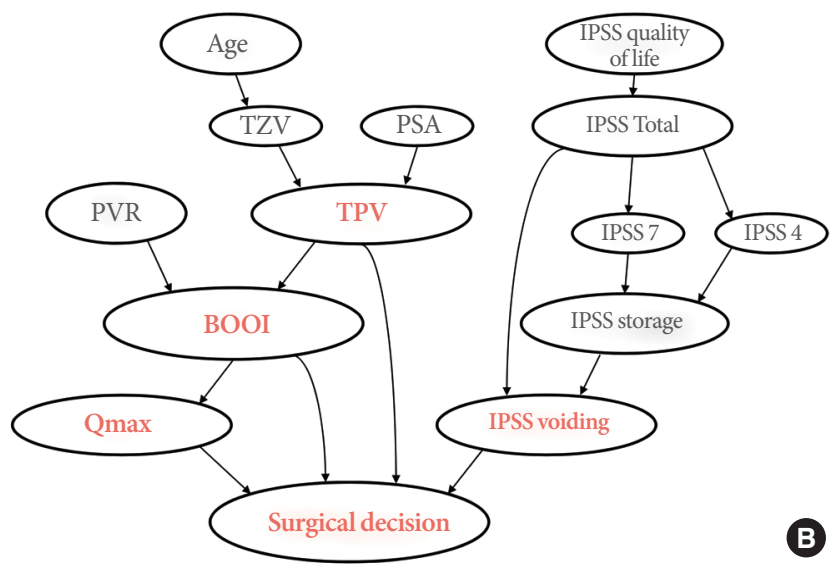

Fig. 1. A causal Bayesian network model for bladder outlet obstruction (A) and surgical decision-making (B), indicating the parameters that directly influenced the presence of urodynamically confirmed bladder outlet obstruction and the physician's surgical decision. These models were established using Banjo version 2.2.0 (Duke University, Durham, NC, USA). TZV, transitional zone volume; PSA, prostatespecific antigen; Qmax, maximal flow rate; PVR, postvoid residual volume; TPV, total prostate volume; BOO, bladder outlet obstruction; IPSS, International Prostate Symptom Score; IPSS voiding subscore, sum of questions 1, 3, 5, and 6 of the IPSS; IPSS storage subscore, sum of questions 2, 4 , and 7 of the IPSS; IPSS quality of life, question 8 of the IPSS; BOOI, bladder outlet obstruction index. 
Table 2. Multivariate logistic regression models for the probability of bladder outlet obstruction

\begin{tabular}{|c|c|c|c|c|c|c|}
\hline \multirow{2}{*}{ Variable } & \multicolumn{3}{|c|}{ All } & \multicolumn{3}{|c|}{ No TPV } \\
\hline & $\mathrm{P}$-value & OR & $95 \% \mathrm{CI}$ & $\mathrm{P}$-value & OR & $95 \% \mathrm{CI}$ \\
\hline Age & 0.78 & 0.997 & $0.978-1.017$ & 0.067 & 1.017 & $0.999-1.035$ \\
\hline Qmax & $<0.001$ & 0.904 & $0.874-0.935$ & $<0.001$ & 0.905 & $0.877-0.933$ \\
\hline PVR & $<0.001$ & 1.002 & $1.002-1.006$ & $<0.001$ & 1.005 & $1.003-1.007$ \\
\hline TPV & $<0.001$ & 1.042 & $1.035-1.050$ & - & - & - \\
\hline
\end{tabular}

OR, odds ratio; CI, confidence interval; Qmax, maximal flow rate; PVR, postvoid residual volume; TPV, total prostate volume.

Table 3. Multivariate logistic regression models for the probability of requiring prostatic surgery

\begin{tabular}{|c|c|c|c|c|c|c|c|c|c|c|c|c|}
\hline \multirow{2}{*}{ Variable } & \multicolumn{3}{|c|}{ All } & \multicolumn{3}{|c|}{ No TPV } & \multicolumn{3}{|c|}{ No BOOI } & \multicolumn{3}{|c|}{ Neither TPV nor BOOI } \\
\hline & P-value & OR & $95 \% \mathrm{CI}$ & P-value & OR & $95 \% \mathrm{CI}$ & P-value & OR & $95 \% \mathrm{CI}$ & P-value & OR & $95 \% \mathrm{CI}$ \\
\hline Age & 0.006 & 1.029 & $1.008-1.051$ & 0.009 & 1.027 & $1.007-1.048$ & $<0.001$ & 1.045 & $1.025-1.066$ & $<0.001$ & 1.045 & $1.026-1.064$ \\
\hline Qmax & $<0.001$ & 0.940 & $0.911-0.969$ & $<0.001$ & 0.911 & $0.884-0.939$ & $<0.001$ & 0.950 & $0.924-0.977$ & $<0.001$ & 0.910 & $0.885-0.935$ \\
\hline PVR & 0.271 & 1.001 & $0.999-1.004$ & 0.047 & 1.002 & $1.000-1.004$ & 0.097 & 1.002 & $1.000-1.004$ & 0.001 & 1.004 & $1.002-1.006$ \\
\hline \multicolumn{13}{|l|}{ IPSS } \\
\hline Voiding subscore & $<0.001$ & 1.065 & $1.032-1.099$ & $<0.001$ & 1.064 & $1.032-1.097$ & $<0.001$ & 1.056 & $1.025-1.088$ & 0.001 & 1.050 & $1.021-1.079$ \\
\hline Storage subscore & 0.002 & 0.924 & $0.879-0.972$ & 0.013 & 0.940 & $0.894-0.987$ & $<0.001$ & 0.917 & $0.874-0.962$ & 0.006 & 0.938 & $0.897-0.981$ \\
\hline Quality of life & 0.002 & 1.265 & $1.088-1.471$ & 0.007 & 1.226 & $1.059-1.419$ & 0.009 & 1.205 & $1.048-1.386$ & 0.033 & 1.152 & $1.011-1.313$ \\
\hline TPV & $<0.001$ & 1.050 & $1.039-1.061$ & $<0.001$ & 1.060 & $1.050-1.070$ & - & - & - & - & - & - \\
\hline BOOI & $<0.001$ & 1.031 & $1.022-1.040$ & - & - & - & $<0.001$ & 1.045 & $1.036-1.053$ & - & - & - \\
\hline
\end{tabular}

TPV, total prostate volume; BOOI, bladder outlet obstruction index; OR, odds ratio; CI, confidence interval; Qmax, maximal flow rate; PVR, postvoid residual volume; IPSS, International Prostate Symptom Score; voiding subscore, sum of questions 1, 3, 5, and 6 of the IPSS; storage subscore, sum of questions 2, 4 , and 7 of the IPSS; quality of life, score of question 8 of the IPSS.

quiring prostatic surgery (Table 3). These variables were used to build the prediction model for both BOO and prostatic surgery. Two formulas were developed to estimate the probability of having $\mathrm{BOO}$ even if TPV is not available. We prepared a total of 4 formulas to assist in surgical decision-making for each of the following situations: all variables present, no TPV, no BOO index, and neither TPV nor BOO index. Using these formulas, nomograms for both $\mathrm{BOO}$ and prostatic surgery were generated using the verified predictors by weighting the relative contribution of the selected variables. We adopted the calculated probability of having BOO to calculate the probability of prostatic surgery if a PFS was not performed. The nomograms are shown in Fig. 2. When the prediction model yielded a calculated probability of having BOO or requiring prostatic surgery of $50 \%$ or more, it was considered that BOO was present or surgery was necessary.

\section{Internal Validation}

A total of 253 male patients with LUTS/BPH were enrolled as an internal validation cohort. There were no significant differences in the IPSS and UFM parameters between the internal validation cohort and the development cohort. However, the mean values of age, TPV, and the BOO index were greater in the internal validation cohort than in the development cohort (Table 1).

In the internal validation cohort, the prediction model was found to have an AUC of 0.816 (95\% confidence interval [CI], 0.764-0.868) for BOO and 0.866 (95\% CI, 0.819-0.914) for the need for surgery (Fig. 3A, C). The sensitivity, specificity, positive predictive value, and negative predictive value were $53.6 \%$, $87.0 \%, 76.6 \%$, and $70.2 \%$ for BOO, and $91.6 \%, 50 \%, 81.6 \%$, and $71.2 \%$ for prostatic surgery, respectively. The calibration plot showed that these prediction models showed a good correspondence between the predicted probabilities of $\mathrm{BOO}$ and the actual rate of urodynamically confirmed BOO (Fig. 3B). In addition, the decision curve showed a high net benefit across the entire spectrum of probability thresholds (Fig. 3C). Regarding the need for prostatic surgery, the calibration plot and the deci- 
Points

Age

Qmax

PVR

TPV

Total points

Linear predictor

Predicted value

Points

Age

Qmax

PVR

Total points

Linear predictor

Predicted value

Points

Age

Qmax

PVR

TPV

IPSS voiding subscore

IPSS storage subscore

IPSS QoL

BOOI

Total points

Linear predictor

Predicted value

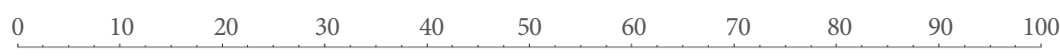

9555

$40 \begin{array}{llllllll}45 & 30 & 25 & 20 & 15 & 10 & 5 & 0\end{array}$

0 50100150200250300350400450500550

\begin{tabular}{lllllllllllll}
\hline 0 & 20 & 40 & 60 & 80 & 100 & 120 & 140 & 160 & 180 & 200 & 220 & 240
\end{tabular}

\begin{tabular}{lllllllllllllll}
\hline 0 & 10 & 20 & 30 & 40 & 50 & 60 & 70 & 80 & 90 & 100 & 110 & 120 & 130 & 140
\end{tabular}

$$
\begin{array}{lllllllllllllll}
\hline-5 & -4 & -3 & -2 & -1 & 0 & 1 & 2 & 3 & 4 & 5 & 6 & 7 & 8
\end{array}
$$

$$
0.1 \quad 0.20 .30 .40 .50 .60 .70 .8 \quad 0.9
$$

(A)

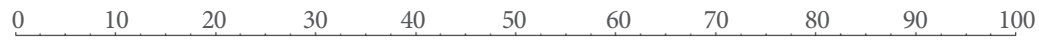

\section{$4 \longdiv { 5 0 5 5 6 0 6 5 7 0 7 5 8 0 8 5 9 0 9 5 }$}

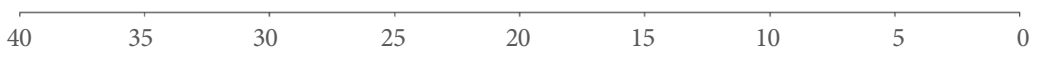

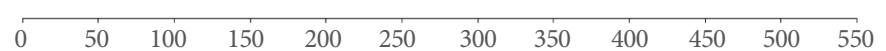

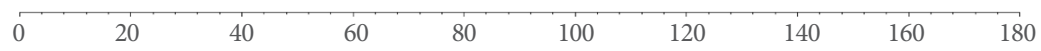

\begin{tabular}{llllllllllllllllll}
\hline-4.5 & -4 & -3.5 & -3 & -2.5 & -2 & -1.5 & -1 & -0.5 & 0 & 0.5 & 1 & 1.5 & 2 & 2.5 & 3
\end{tabular}

$$
\begin{array}{lllllllll}
0.1 & 0.2 & 0.3 & 0.4 & 0.5 & 0.6 & 0.7 & 0.8 & 0.9
\end{array}
$$

\begin{tabular}{|c|c|c|c|}
\hline 0 & 10 & & \\
\hline
\end{tabular}

B

\section{0}

40 \begin{tabular}{l}
1 \\
\hline
\end{tabular} 302520151050

0150350550

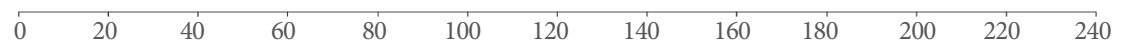

$\begin{array}{lllll:l}0 & 4 & 8 & 12 & 1620\end{array}$

$16128+40$

$$
\begin{array}{lllll}
1 & 3 & 5 \\
0 & 2 & 4 & 4 & 4
\end{array}
$$

$\begin{array}{lllllllllllll}-40 & -20 & 0 & 20 & 40 & 60 & 80 & 100 & 120 & 140 & 160 & 180 & 200\end{array}$

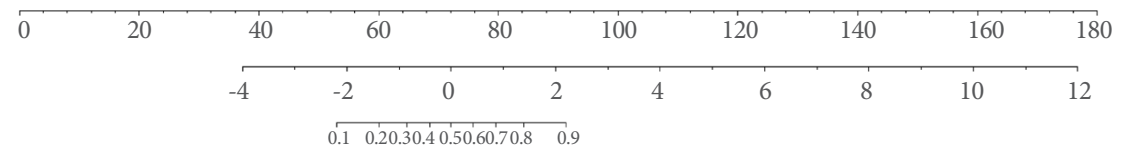

C

Fig. 2. Nomograms predicting urodynamically confirmed BOO and the need for prostatic surgery: (A) for BOO with all parameters, (B) for $\mathrm{BOO}$ without the total prostate volume, (C) for prostatic surgery with all parameters. (Continued to the next page) 
Points

Age

Qmax

PVR

TPV

IPSS voiding subscore

IPSS storage subscore

IPSS QoL

Total points

Linear predictor

Predicted value

Points

Age

Qmax

PVR

IPSS voiding subscore

IPSS storage subscore

IPSS QoL

BOOI

Total points

Linear predictor

Predicted value

Points

Age

Qmax

PVR

IPSS voiding subscore

IPSS storage subscore

IPSS QoL

Total points

Linear predictor

Predicted value

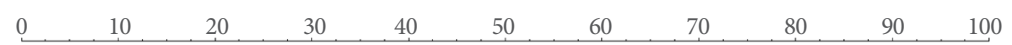

$45^{\prime \prime 25}+105$

$\begin{array}{llllllllll}40 & 35 & 30 & 25 & 20 & 15 & 10 & 5 & 0\end{array}$

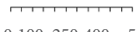

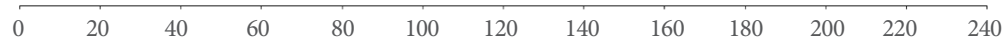

$\begin{array}{llllll}0 & 4 & 8 & 1216 & 120\end{array}$

$161284+40$

$\begin{array}{llll}1 & 3 & 5 \\ 1 & 1 & 5 \\ 0 & 2 & 4 & 4\end{array}$

\begin{tabular}{lllllllllll}
\hline 0 & 20 & & 40 & 60 & & 80 & 100 & 120 & 140 & 160 \\
\cline { 2 - 9 } & -4 & -2 & 0 & 2 & 4 & 6 & 8 & 10 & 12
\end{tabular}

$0.1 \quad 0.20 .30 .40 .50 .60 .70 .8 \quad 0.9$

D

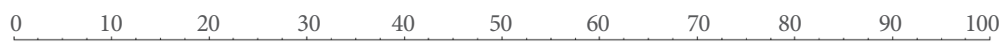

$\begin{array}{llllllll}45 & 55 & 65 & 7 & 75 & 85 & 95\end{array}$

$40 \lcm{15302520151050}$

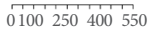

$0+4 \quad 8+121620$

161286420

$\underset{0}{1}+\frac{3}{2}+\frac{5}{4}+\frac{1}{4}$

\begin{tabular}{rrrrrrrrrrrrr}
\hline-40 & -20 & 0 & 20 & 40 & 60 & 80 & 100 & 120 & 140 & 160 & 180 & 200
\end{tabular}

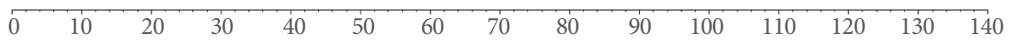

\begin{tabular}{llllllllllll}
\hline-4 & -3 & -2 & -1 & 0 & 1 & 2 & 3 & 4 & 5 & 6 & 7
\end{tabular}

$\begin{array}{llllllll}0.1 & 0.2 & 0.3 & 0.4 & 0.50 .6 & 0.7 & 0.8 & 0.9\end{array}$

E

$\begin{array}{llllllllll}0 & 10 & 20 & 30 & 40 & 50 & 60 & 70 & 80 & 90\end{array}$

$\begin{array}{lllllllllll}45 & 50 & 55 & 60 & 65 & 70 & 75 & 80 & 85 & 90 & 95\end{array}$

\begin{tabular}{llllllll}
\hline 40 & 35 & 30 & 25 & 20 & 15 & 1 & 1
\end{tabular}

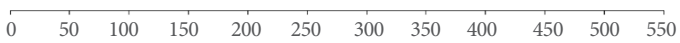

\begin{tabular}{lllllllllll}
\hline 0 & 2 & 4 & 6 & 8 & 10 & 12 & 14 & 16 & 18 & 20
\end{tabular}

$\begin{array}{lllllllll}16 & 14 & 12 & 10 & 8 & 6 & 4 & 2 & 0\end{array}$

$\begin{array}{lllll}1 & 3 & 5 & \\ 0 & 2 & 4 & 6\end{array}$

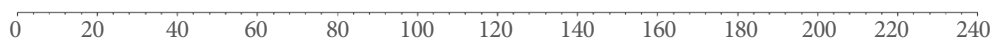

\begin{tabular}{llllllllllllll}
\hline-3 & -2.5 & -2 & -1.5 & -1 & -0.5 & 0 & 0.5 & 1 & 1.5 & 2 & 2.5 & 3 & 3.5
\end{tabular}

$\begin{array}{lllllllll}0.1 & 0.2 & 0.3 & 0.4 & 0.5 & 0.6 & 0.7 & 0.8 & 0.9\end{array}$

F

Fig. 2. (Continued) (D) for prostatic surgery without the BOO index, (E) for prostatic surgery without the total prostate volume, (F) for prostatic surgery without the BOO index and total prostate volume. BOO, bladder outlet obstruction; Qmax, maximal flow rate; PVR, postvoid residual volume; TPV, total prostate volume; IPSS, International Prostate Symptom Score; IPSS voiding subcore, sum of questions 1,3, 5, and 6 of the IPSS; IPSS storage subscore, sum of questions 2, 4 , and 7 of the IPSS; IPSS quality of life, score of question 8 of the IPSS; BOOI, bladder outlet obstruction index. 

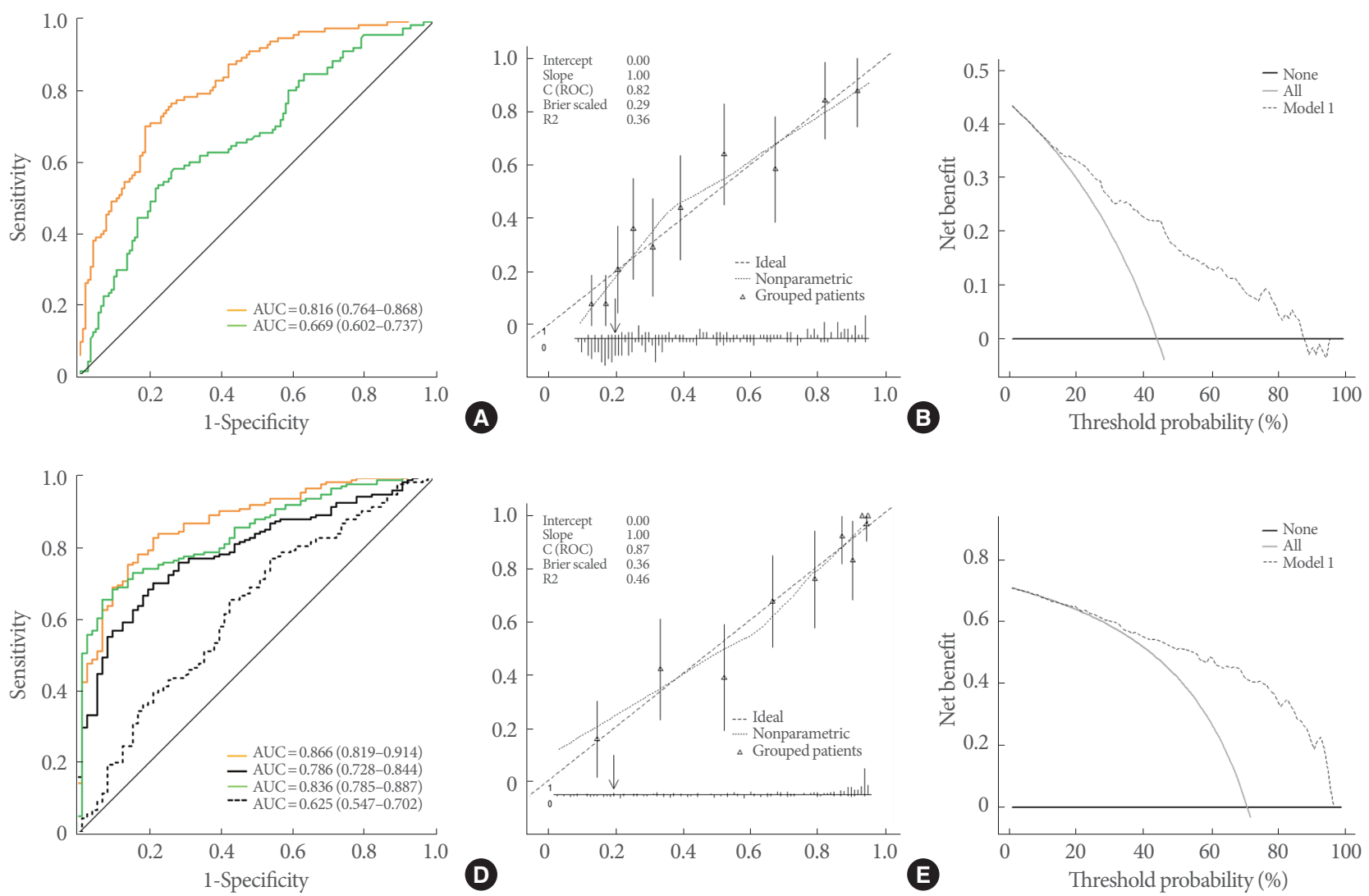

Fig. 3. Internal validation of the nomogram predicting urodynamic bladder outlet obstruction and prostatic surgery. (A) Receiver operating characteristic (ROC) curve of the nomogram for bladder outlet obstruction; the orange line indicates the nomogram with all parameters, and the green line indicates the nomogram without the bladder outlet obstruction (BOO) index. (B) A calibration plot to show the predicted probability of bladder outlet obstruction. (C) Decision curve analysis revealed that the prediction model for bladder outlet obstruction provided a superior net benefit. (D) ROC curve of the nomogram for prostatic surgery; the orange line indicates the nomogram with all parameters, the black line indicates the nomogram without the $\mathrm{BOO}$ index, the green line indicates the nomogram without total prostate volume, and the black dotted line indicates the nomogram without the $\mathrm{BOO}$ index and total prostate volume. (E) A calibration plot to show the predicted probability of prostatic surgery. (F) Decision curve analysis revealed that the prediction model for the need for prostatic surgery provided a superior net benefit.

sion curve likewise showed a good correspondence and a high net benefit (Fig. 3E, F).

\section{DISCUSSION}

We conducted this study using a large database of urodynamic studies to develop formulas to predict $\mathrm{BOO}$ and the need for prostatic surgery. These formulas were calculated based on the urodynamic evidence of BOO. We developed 4 formulas, some of which can be used even when 1 or 2 parameters are not available. To the best of our knowledge, this is the first study of the development of a BOO-based decision-support prediction model for prostatic surgery in patients with LUTS/BPH.
Histologically, BPH corresponds to a proliferation of the stromal and epithelial cells in the prostate with aging. However, clinically, it is a heterogeneous condition composed of different aspects of LUTS, benign enlargement of the prostate, and functional BOO. It is well known that there are poor correlations among patients in terms of LUTS, BOO, and prostate volume [11]. Although a patient's symptom severity or quality of life is important, these subjective factors are not absolutely reflective of objectively measurable parameters. For example, patients with detrusor underactivity without either prostate enlargement or BOO can have significant symptoms and bother. Therefore, a surgical decision based simply on the prostate volume or subjective LUTS might be inappropriate. 
In patients with marginal clinical situations, urodynamically confirmed BOO can be used as an important guide in surgical decision-making. Urodynamic studies have been advocated as a useful predictor of the success of surgery, and this has been encouraged by international guidance [12,13]. Our meta-analysis likewise showed a significant association between urodynamically confirmed $\mathrm{BOO}$ and greater improvements in all treatment outcome parameters. Preoperative urodynamic studies may add insight into postoperative outcomes after the surgical treatment of $\mathrm{BPH}$ [14]. BOO is significantly associated with both functional and morphological alterations of the urinary bladder. Animal experiments have demonstrated that chronic $\mathrm{BOO}$ can cause repeated bladder ischemia/reperfusion injuries, ultimately resulting in partial denervation of the bladder $[15,16]$. Such oxidative stress is responsible for bladder hyperactivity in moderate cases or detrusor underactivity, if it is prolonged $[17,18]$. Abundant clinical evidence has shown that $\mathrm{BOO}$ is significantly associated with functional changes in the urinary bladder. We analyzed the clinical and urodynamic characteristics of 429 elderly men with LUTS/BPH according to a urodynamic study. Patients with BOO had a significantly higher rate of involuntary detrusor contraction and poor compliance compared to the patients without BOO [19]. Therefore, it is functional $\mathrm{BOO}$ that directly causes undesirable consequences, such as overactive bladder or underactive bladder. In addition, classic findings have also shown that urodynamically confirmed BOO is closely associated with morphological changes of the urinary bladder. A significant correlation was found between bladder trabeculation and the grade of $\mathrm{BOO}$ in old men with presumptive LUTS/BPH [20,21]. BOO confirmed through a urodynamic study was also closely related with radiologically demonstrated trabeculation [21]. Therefore, bladder dysfunction secondary to $\mathrm{BOO}$ must be considered in the management of male patients with LUTS. The surgical treatment of patients who satisfy the absolute indications for surgery is often too late. This means that the conditions might be irreversible, in functional or morphological aspects, even after surgical relief.

In patients who do not meet the absolute indications for surgery, patient selection for surgery is often not easy. This decision may depend on the degree of bother of the LUTS, prostate volume, comorbidities, a patient's personal preferences, the financial status of the patient, the patient's willingness to accept potential surgery-associated complications, the experience of the surgeon, and the availability of a specific surgical armamen- tarium. In our institution, the decision to perform surgery in patients who did not meet the absolute indications was made more based on the presence of urodynamically confirmed BOO than on patient requests or prostate volume. We do not commonly recommend surgery to patients when they do not have significant $\mathrm{BOO}$, even though the patient may have an enlarged prostate. PFS is considered as the gold standard for diagnosing BOO. Unfortunately, none of the noninvasive tests for diagnosing $\mathrm{BOO}$ in men with LUTS can currently be recommended as an alternative to a PFS [22]. However, urodynamic studies are not recommended for routine clinical use due to their invasiveness, expense, time-consuming nature, and technical challenges [23]. For this reason, we thought that a prediction model for BOO-based surgical decision-making without performing PFS would be very important. This study is an extension of our previous clinical studies involving BOO in patients with LUTS/BPH. We have performed several clinical studies using data that we have accumulated over the last 15 years. We sought to identify noninvasive parameters for predicting BOO using our clinical data, and found that Qmax played a significant role in predicting $\mathrm{BOO}$ in Korean men with LUTS [19]. More recently, we also analyzed data from 1,381 patients with $\mathrm{BPH}$ using $\mathrm{CBN}$ analysis and found that TPV, Qmax, and PVR were independent predictive parameters of urodynamically confirmed BOO [8].

One of the advantages of our urodynamic data is that the urodynamic studies were performed by the same investigators in a single center using the same practice protocol since 2002 when the International Continence Society standardization was published [5]. In our institution, the prevalence of $\mathrm{BOO}$ among patients with LUTS/BPH is relatively high. We have a high number of patients who potentially need prostate surgery, as our hospital is a tertiary referral center with a heavy clinical volume. In this dataset, patients with an absolute indication for prostatic surgery were also included. This means that the clinical parameters of these patients were incorporated in these formulas. If patients did not agree to undergo surgery when the clinician recommended it, we still classified these patients as requiring prostatic surgery. The category of requiring surgery in this study solely reflects the decisions made by urologists.

A major limitation of our study is that only a few surgeons were involved in surgical decision-making in the $\mathrm{BPH}$ population. This might also have caused some bias in the surgical decisions, making these results not generalizable. However, the need for surgery determined by the surgeons is not essentially 
different from what would be expected based on textbook guidelines, since they followed the clinical guidelines published by the American Urological Association or the European Urological Association in their routine clinical practice $[2,3]$. Additionally, the need for surgery was not merely judged by the patient's request, but by considering the entire clinical situation of the patients, including more objective parameters such as urodynamically confirmed BOO. We believe that surgical decisionmaking in real clinical situations for LUTS/BPH patients whose conditions do not satisfy the absolute indications for surgery should be primarily based on the presence of functional obstruction. The purpose of surgery in patients with LUTS/BPH is to relieve conditions such as acute urinary retention, gross hematuria of prostatic origin, associated bladder stones, or hydronephrosis in patients who satisfy the absolute surgical indications for BPH. However, symptom improvement and overall patient satisfaction after prostate surgery are primary goals in patients who do not satisfy the absolute surgical indications. Based on our unpublished data, we found that the overall patient satisfaction after prostatectomy in patients with LUTS/ BPH was very high (over 90\%), with the current surgical decision-making process being based mainly on urodynamically confirmed BOO. We believe that our current process of selecting patients for surgery is reasonably justified.

We expect that this urodynamic BOO-based prediction model will be of great help in actual clinical practice. We believe that further validation in other ethnic populations is necessary, as this formula is based on an Asian population. Additionally, more research into subjective satisfaction and symptom improvement in patients who undergo prostatic surgery based on this nomogram is needed to justify our work.

In conclusion, we established nomograms for the prediction of $\mathrm{BOO}$ and the need for BOO-related surgery in patients with LUTS/BPH. Internal validation of the nomograms demonstrated that these nomograms predicted both $\mathrm{BOO}$ and requiring prostatic surgery very well. We hope that these $\mathrm{BPH}$ probability models will help clinicians to better select LUTS/BPH patients requiring surgical treatment.

\section{ACKNOWLEDGEMENTS}

Kwi-Shik Kim and Yu-Kyung Lee assisted with database management.

\section{REFERENCES}

1. Choo MS, Han JH, Shin TY, Ko K, Lee WK, Cho ST, et al. Alcohol, smoking, physical activity, protein, and lower urinary tract symptoms: prospective longitudinal cohort. Int Neurourol J 2015;19:197206.

2. Gratzke C, Bachmann A, Descazeaud A, Drake MJ, Madersbacher $S$, Mamoulakis $C$, et al. EAU guidelines on the assessment of nonneurogenic male lower urinary tract symptoms including benign prostatic obstruction. Eur Urol 2015;67:1099-109.

3. McVary KT, Roehrborn CG, Avins AL, Barry MJ, Bruskewitz RC, Donnell RF, et al. Update on AUA guideline on the management of benign prostatic hyperplasia. J Urol 2011;185:1793-803.

4. Jeon HJ, Choo MS, Oh SJ. The effect of posture and repetition on urodynamic parameters: a prospective randomized study. Investig Clin Urol 2017;58:34-41.

5. Abrams P, Cardozo L, Fall M, Griffiths D, Rosier P, Ulmsten U, et al. The standardisation of terminology of lower urinary tract function: report from the Standardisation Sub-committee of the International Continence Society. Neurourol Urodyn 2002;21:167-78.

6. Lim CS, Abrams P. The Abrams-Griffiths nomogram. World J Urol 1995;13:34-9.

7. Kim M, Ramirez L, Yoo C, Choo M, Paick JS, Oh SJ. Factors influencing nonabsolute indications for surgery in patients with lower urinary tract symptoms suggestive of benign prostatic hyperplasia: analysis using causal bayesian networks. Int Neurourol J 2014;18: 198-205.

8. Kim M, Cheeti A, Yoo C, Choo M, Paick JS, Oh SJ. Non-invasive clinical parameters for the prediction of urodynamic bladder outlet obstruction: analysis using causal Bayesian networks. PLoS One 2014;9:e113131.

9. Hosmer DW, Lemeshow S. Assessing the fit of the model. In: Hosmer DW, Lemeshow S. Applied logistic regression. 2nd ed. New York: John Wiley \& Sons; 2005. p. 143-202.

10. Vickers AJ, Elkin EB. Decision curve analysis: a novel method for evaluating prediction models. Med Decis Making 2006;26:565-74.

11. Lepor H. Evaluating men with benign prostatic hyperplasia. Rev Urol 2004;6 Suppl 1:S8-15.

12. Thomas AW, Cannon A, Bartlett E, Ellis-Jones J, Abrams P. The natural history of lower urinary tract dysfunction in men: the influence of detrusor underactivity on the outcome after transurethral resection of the prostate with a minimum 10-year urodynamic follow-up. BJU Int 2004;93:745-50.

13. Thiruchelvam N, Drake MJ, Venn S, Morley R; BAUS section of Female, Neurological and Urodynamics. A 2014 snapshot audit of 
the role of urodynamics in the UK for benign prostatic enlargement surgery. Neurourol Urodyn 2016;35:271-2.

14. Kim M, Jeong CW, Oh SJ. Diagnostic value of urodynamic bladder outlet obstruction to select patients for transurethral surgery of the prostate: Systematic review and meta-analysis. PLoS One 2017;12: e0172590.

15. Greenland JE, Brading AF. The effect of bladder outflow obstruction on detrusor blood flow changes during the voiding cycle in conscious pigs. J Urol 2001;165:245-8.

16. Nomiya M, Sagawa K, Yazaki J, Takahashi N, Kushida N, Haga N, et al. Increased bladder activity is associated with elevated oxidative stress markers and proinflammatory cytokines in a rat model of atherosclerosis-induced chronic bladder ischemia. Neurourol Urodyn 2012;31:185-9.

17. Yamaguchi O, Nomiya M, Andersson KE. Functional consequences of chronic bladder ischemia. Neurourol Urodyn 2014;33:54-8.

18. Nomiya M, Yamaguchi O, Akaihata H, Hata J, Sawada N, Kojima Y, et al. Progressive vascular damage may lead to bladder underactivi- ty in rats. J Urol 2014;191:1462-9.

19. Kang MY, Ku JH, Oh SJ. Non-invasive parameters predicting bladder outlet obstruction in Korean men with lower urinary tract symptoms. J Korean Med Sci 2010;25:272-5.

20. el Din KE, de Wildt MJ, Rosier PF, Wijkstra H, Debruyne FM, de la Rosette JJ. The correlation between urodynamic and cystoscopic findings in elderly men with voiding complaints. J Urol 1996;155: 1018-22.

21. Shah PJ, Whiteside CG, Milroy EJ, Turner-Warwick RT. Radiological trabeculation for the male bladder--a clinical and urodynamic assessment. Br J Urol 1981;53:567-70.

22. Gravas S, Bach T, Drake M, Gacci M, Gratzke C, Hermann TR, et al. Treatment of non-neurogenic male LUTS [Internet]. Anheim (NL): European Association of Urology, c2017 [cited 2017 Mar 22]. Available from: http://uroweb.org/guideline/treatment-of-nonneurogenic-male-luts/.

23. D’Ancona CA, Bassani J, Almeida JC. Noninvasive urodynamic evaluation. Int Neurourol J 2012;16:116-21. 